\title{
INCIDENCE OF HEMIDIAPHRAGMATIC PARALYSIS WITH PATIENT CONTROLLED INFUSION OF LOW VOLUME OF ROPIVACAINE AFTER USG GUIDED LOW DOSE INTERSCALENE BRACHIAL PLEXUS BLOCK, A PROSPECTIVE OBSERVATIONAL STUDY
}

\author{
Dr Tanvir Samra, Dr Pankaj Kushal, Dr Vishal Kumar \\ Postgraduate Institute Of Medical Education And Research (PGIMER),Chandigarh, India
}

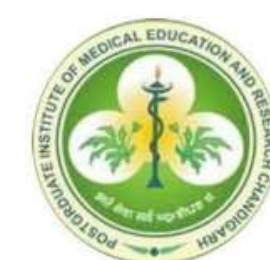

INTRODUCTION:Diaphragmatic paresis due to phrenic nerve block is a side-effect of interscalene brachial plexus block (ISB).

-It is administered for anaesthesia and analgesia in patients with fractures of the humerus and injuries to the shoulder joint.

-USG guided ISB increase success rate and minimise complications.

- Continuous blocks are favoured over single shot to minimise supplemental opioid requirements, improves sleep quality and increases patient satisfaction

AIM-To assess the incidence of hemidiaphragmatic paralysis in patients administered low dose patient controlled interscalene analgesia $(2 \mathrm{ml} / \mathrm{hr}$ of $0.2 \%$ ropivacaine) after USG guided low dose bolus injection of $10 \mathrm{ml}$ of $0.5 \%$ ropivacaine.

\section{MATERIALS AND METHODS}

-Approved by ethics and registered on clinicaltrials.gov (NCT03081728) -Prospective, randomised, observational study ( 2016-2017)

-Inclusion criteria: Patients aged 18-65 years belonging to ASA PS I-III with either fractures of proximal humerus or shoulder dislocations

-Exclusion criteria: Patient's refusal for block, allergy to amide local anaesthetic, inability to operate PCA and interpret VAS, coagulopathy, severe respiratory disease $\left(\mathrm{FEV}_{1} / \mathrm{FVC}<0.7\right.$ and $\mathrm{FEV}_{1}<80 \%$ of predicted), infection at the block site, prior history of neck surgery or radiation to the neck, neurological deficit in the arm or suspicion of nerve injury, patients with polytrauma or head injury $(\mathrm{GCS}<15)$ and chronic opioid abusers

-Non-random allocation based on the post-operative analgesic modality employed:

\section{-Group I: Patient controlled interscalene analgesia (PCIA)}

-Group II: Multimodal analgesia (MA)-Acetaminophen (1 gm) and diclofenac $75 \mathrm{gm}$ (rescue tramadol) was administered.

- Pre-procedural ultrasound scanning:To_measure diaphragmatic excursion - General Anesthesia Protocol: Propofol, morphine, vecuronium for induction. O2/N2O/Isoflurane (1 MAC) for maintanence of anaesthesia - Assessment of pain: No intervention was done for patients with VAS $<3$. $V A S>3$

*Patients in Group I (PCIA) administered $10 \mathrm{ml}$ of $1 \%$ lignocaine *Patients in Group II administered fentanyl bolus dose of $0.5 \mu \mathrm{g} / \mathrm{kg}$. $V A S$ re-assessed after 20 minutes and if VAS >3

*Patients in Group I managed with $2^{\text {nd }}$ dose of $10 \mathrm{ml}$ of $1 \%$ lignocaine * Patients in Group II administered a second fentanyl bolus dose $0.5 \mu \mathrm{g} / \mathrm{kg}$. The patients were monitored for the first 24 hours

RESULTS: Incidence of complete and partial paresis of diaphragm after extubation was $85 \%$ and $3.7 \%$. Mean value of diaphragmatic excursion was $1.5 \pm 0.8 \mathrm{~cm}(1-1.9 \mathrm{~cm})$ in patients with paresis

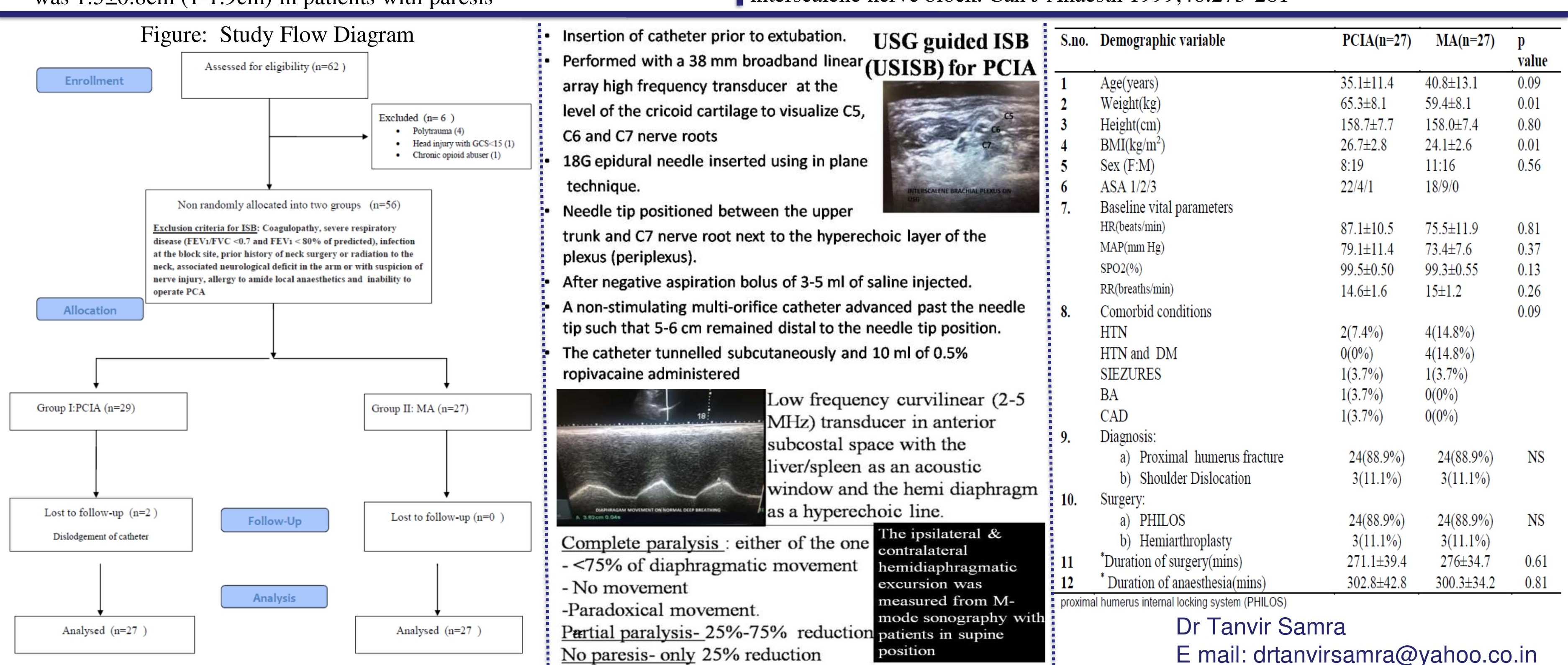

RESULTS (contd...)

-Single PCA bolus dose administered by 13 patients $(48 \%)$ in the first 6 hours

- Two bolus doses/hour administered by 2 patients in first 6 hours and only one patient took two bolus doses/hour in the next 6 hours. -Partial paresis in all the six instances in which two bolus doses/hour administered but diaphragmatic excursion normalized in the next recording made at 3 hours. No paresis in patients administered background infusion/single bolus doses of ropivacaine and scanned at 30 minutes after bolus and at 4, 6,12 and $24 \mathrm{hrs}$.

- No episodes of vomiting, respiratory discomfort, Horner's syndrome pneumothorax, LAST or intravascular injections

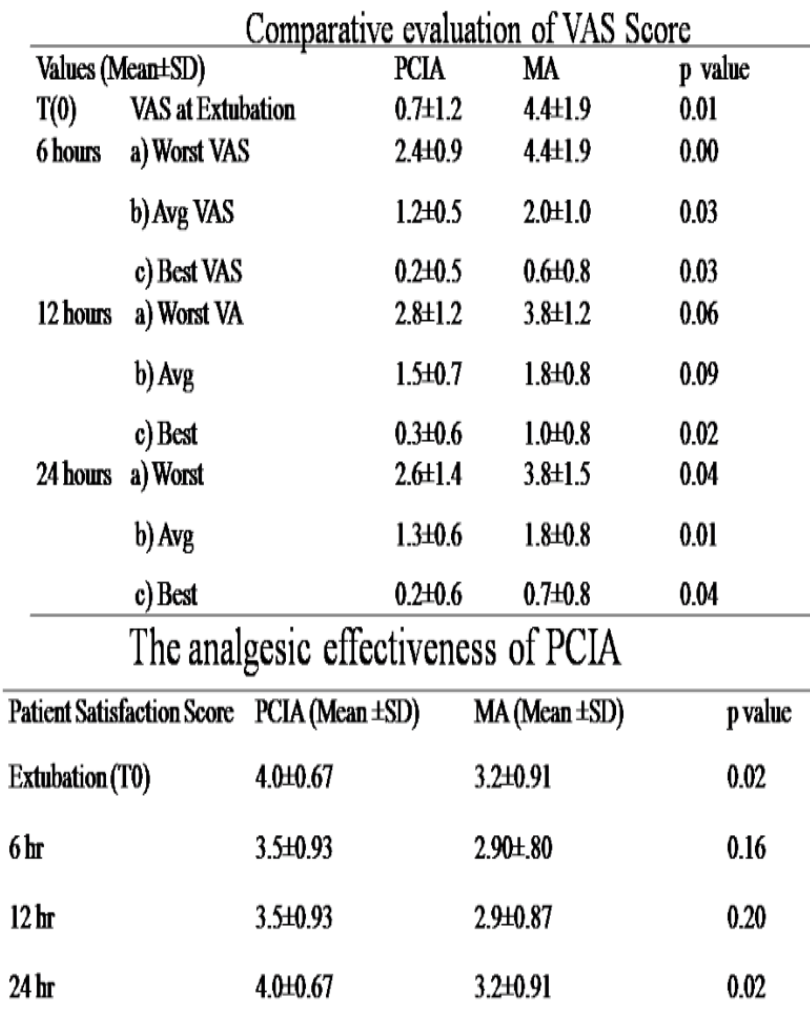

DISCUSSION: Single shot ISB impair pulmonary function and continuous infusions have not been used by clinicians with the hypothesis that continuous ISB would prolong pulmonary dysfunction. $3.6 \mathrm{ml}$ is the minimum effective volume of local anaesthetic ( $0.75 \%$ ropivacaine) for shoulder analgesia in $95 \%$ patients using US-guidance at the C7 root level. This dose does not lead to hemidaipahragmatic paralysis as measured by USG.Limitations of our study were that blinding and randomisation was not done as only the eligible patients were allocated the block group.Comparision of diaghragmatic excursion in controls was not done. In this study the potential for inter observer variability in distance measurements using on-screen caliper tool was

not excluded as evaluation of the reproducibility of these measurements was not done. Moreover results of this study relate only to ISB performed for analgesic purposes and may not apply for surgical analgesia.

CONCLUSION:Ultrasound guided interscalene brachial plexus block at C6-C7 root using $10 \mathrm{ml}$ of $0.5 \%$ ropivacaine causes diaphragmatic paresis in $85 \%$ patients, with no clinical implications. Continuous infusion of ropivacaine $0.2 \%$ at $2 \mathrm{ml} / \mathrm{hr}$ with on demand $5 \mathrm{ml}$ boluses administered via an ultrasound-guided C6-C7 root/superior trunk perineural catheter produces effective analgesia and high Patient Satisfaction Score and no hemidiaphragmatic paralysis.

\section{REFERENCES}

1. McNaught A, Shastri U, Carmichael N, Awad I, Columb M, Cheung J et al. Ultrasound reduces the minimum effective local anaesthetic volume compared with peripheral nerve stimulation for interscalene block. Br. J Anaesth 2011;106:124-130. 2.Renes S, van Geffen G, Rettig H, Gielen M, Scheffer G. Minimum Effective Volume of Local Anesthetic for Shoulder Analgesia by Ultrasound-Guided Block at Root C7 With Assessment of Pulmonary Function. Reg Anesth and Pain Med. 2010;35:529-534.

3.Boezaart A, de Beer J, du Toit C, van Rooyen K. A new technique of continuous interscalene nerve block. Can J Anaesth 1999;46:275-281 